\title{
émulations
}

\section{Michel Agier - Anthropologie de la ville}

\section{Laurie Daffe}

Émulations - Revue de sciences sociales

2015, «Comptes rendus critiques, En ligne »

\section{Article disponible à l'adresse suivante}

https://ojs.uclouvain.be/index.php/emulations/article/view/7063

\section{Pour citer cet article}

Laurie Daffe, « Michel Agier - Anthropologie de la ville », Émulations, en ligne. Mise en ligne le 21 septembre 2015.

DOI : 10.14428/emulations.cr.010

Distribution électronique : Université catholique de Louvain (Belgique) : ojs.uclouvain.be

(C) Cet article est mis à disposition selon les termes de la Licence Creative Commons Attribution, Pas d'Utilisation Commerciale 4.0 International. http://creativecommons.org/licenses/by-nc/4.0/

Éditeur : Émulations - Revue de sciences sociales / Presses universitaires de Louvain https://ojs.uclouvain.be/index.php/emulations

ISSN électronique : 1784-5734

UCL PRESSES 


\section{Michel Agier - Anthropologie de la ville}

Laurie Daffe ${ }^{1}$

Recensé: Michel Agier, Anthropologie de la ville, Paris, Presses universitaires de France, 2015.

Alors que Claude-Levi Strauss considérait la ville comme le lieu d'une « ethnographie du dimanche » (p. 22), Michel Agier s'emploie à nous démontrer dans cet ouvrage que la ville est désormais le lieu de l'anthropologie. Pour autant, le lecteur n'y trouvera pas de définition préétablie de la ville : loin d'être un donné, elle y est décrite comme une totalité invisible et décomposée, inexorablement vouée au changement ; un «hologramme » reconstruit par chacun (Agier, 2015). Selon l'auteur, il n'en reste pas moins possible de l'approcher conceptuellement, mais aussi d'en réécrire l'anthropo-logique, « en partant du point de vue des pratiques, des relations et des mots des citadins tels que le chercheur lui-même les observe et les note, directement en situation » (p. 25). Agier l'illustre en partageant ses observations de terrain à Lomé (Togo) et à Salvador de Bahia (Brésil) afin de développer les pistes théoriques, empiriques et méthodologiques (voire politiques) qu'il propose.

En effet, de nombreux thèmes et exemples chers à l'auteur sont approfondis tout au long des trois parties qui structurent l'ouvrage, elles-mêmes subdivisées en trois chapitres. Michel Agier débute avec « La ville des anthropologues », dans laquelle il retrace le parcours de l'ethnologue en ville. Une expérience qui s'avère être à la fois fragmentée et sensible, conduisant l'auteur à présenter la ville comme un « hologramme » (chapitre I). Les concepts de région, de situation et de réseau ainsi que l'approche situationnelle (Clyde Mitchell, Ulf Hannertz) permettent ensuite d'investiguer ce parcours d'un point de vue aussi bien conceptuel que méthodologique (chapitres II \& III). Dans la seconde partie de l'ouvrage («La ville à l'œuvre »), Michel Agier analyse la manière dont la ville est produite et transformée - symboliquement et matériellement — par la familiarité et l'engagement de chacun (chapitre IV). Ces manières de «faire ville » sont multiples, mais c'est en partant de l'étude des lieux, des non-lieux et des hors-lieux (chapitre V) que l'auteur examine plus particulièrement un exemple de commencement de ville : celui des camps et des zones de transit (chapitre VI). L'ouvrage se conclut sur « La ville en mouvements », troisième partie consacrée aux conditions de l'agir urbain et à la ville en tant que dispositif culturel. Y sont respectivement abordées des thématiques aussi

${ }^{1}$ Doctorante FRESH (FNRS), Laboratoire d'Anthropologie Prospective (Université catholique de Louvain). 
fondamentales que les moments de création culturelle ("performances»), l'invention des identités urbaines et les mouvements de rue (chapitre VII) ; les rassemblements politiques et plus précisément les communautés de l'instant, de rencontres et de mouvement (chapitre VIII); enfin, les occupations, les invasions et les installations (chapitre IX). Autant de mouvements qui sont à la fois prises d'espace et prises de parole, et qui «peuvent changer la distribution des espaces et le sens de la ville en même temps qu'ils "font" la ville » (p. 207), nous dit Agier.

Selon nous, c'est dans cette dernière affirmation, qui structure le mobile général de l'ouvrage, ainsi que dans les thèses transversales qui le parcourent, que résident l'intérêt autant que la nécessité de cette Anthropologie de la ville. Tout d'abord, l'auteur y soutient la légitimité de la place de l'anthropologue en ville. Celui-ci est amené à y explorer " ce qui "fait ville" sur le plan des relations sociales, de la symbolique des espaces, et de leur édification matérielle»(p.9). C'est ce «faire ville», véritable mise en œuvre du « droit à la ville » d'Henri Lefebvre, qui lie les différentes parties de l'ouvrage. Grâce aux apports de l'École de Chicago, de l'École de Manchester et de l'anthropologie française, la problématique glisse donc de la recherche de l'essence de la ville (ce qu'elle « est ») à la description des processus par lesquels les individus et les groupes en font des espaces vécus, partagés, caractérisés par la complexité, le mouvement et la relation. L'anthropologue est donc invité à chercher partout les possibilités de commencement de la ville et de ce qui y naît. Partout, car « l'anthropologie de la ville est sans bornes, "tout terrain" » (p. 33).

Toutefois, pour l'auteur, ces questionnements gagnent à être étudiés à partir de deux cadres spatiaux en particulier : l'échelle planétaire et les marges. La première est définie comme «le nouveau tout où se déploient les problématiques les plus ordinaires et quotidiennes de la vie en société » (p. 32). Par là, Michel Agier place la ville au sein d'un réseau plus vaste de «lieux de la mobilité » (p. 30) dans lequel elle prend son sens. En effet, si tous les chemins mènent à la ville, alors la mobilité et le « dé-placement » (p. 30), deviennent les facteurs centraux de sa création mais aussi de sa transformation. Et de souligner ainsi la tension permanente entre mobilité et immobilité traduite dans le processus du «faire-ville » (p. 152) : « y sont à l'œuvre le besoin de fondation d'un lieu pour tout humain, comme le besoin d'ancrage pour des vies plus mobiles » (p. 215). Du « racinement » se crée alors dans les marges, les frontières et les interstices. Squats, cités de transit, ghettos, refuges, installations, invasions... sont les corollaires localisables de ces mouvements. L'auteur coupe court à la critique facile de l'évidente relativité de ce qu'il entend désigner : il n'y a ni centre, ni marge en soi, l'un et l'autre n'existent qu'en relation. Mais, loin de se contenter de ce constat, Michel Agier en fait au contraire un argument supplémentaire en faveur d'un nécessaire " dé-centrement » : c'est précisément dans cette relation que se jouent les enjeux des lieux.

En effet, face à une extension urbaine illimitée, la centralité dispose d'une force et d'une capacité de résistance qui tiennent de ce qu'elle attire ou rejette et donc, a fortiori, n'existe que dans une relation permanente et dynamique avec un pourtour, un dehors. 
«Les hors-lieux se constituent d'abord comme des dehors, placés sur les bords ou les limites de l'ordre normal des choses - ordre "normal" qui reste jusqu'aujourd'hui, en fin de compte, un ordre national et toujours plus urbain. » (p.138) On parle donc bien d'une anthropologie du ban-lieu, de mises à l'écart autant politiques que territoriales. En-dehors de la ville officielle «montrée et aménagée », ces espaces de vie «émergent en creux [...] sur une base inverse de celle de la ville conçue comme espace plein » (p. 154).

C'est pour cette raison que Michel Agier considère les « hors-lieux » comme de véritables «terrains d'innovation anthropologique» (p. 59), en ce qu'ils font bouger « les frontières de l'ordre social et urbain » (p. 30), déplacent progressivement les centres et «modifient les cadres de la réflexion sur les identités locales et sur la formation d'une partie au moins des futurs espaces urbains » (p. 144). En effet, c'est ainsi, nous dit-il, que seront les villes de demain : réversibles, modelables, transposables; « des villes en kit, aux infrastructures minimalistes et mouvantes » (p. 142).

À travers ses hypothèses, Michel Agier avance un double positionnement : d'une part, la nécessité d'une « mise à plat égalitaire du statut théorique de toutes les formes urbaines dans le monde » (p. 33). L'inégalité urbaine-globale en la matière peine en effet à se dégager du prisme de la ville européenne, longtemps érigée en étalon. D’autre part, et dans la continuité de cette idée, l'auteur plaide en faveur d'un indispensable renouvellement des outils de l'anthropologie face aux changements dont elle est contemporaine - mobilités, croissance urbaine rapide, diversification des formes d'agglomération des hommes sur la planète (p.16). Autant d'évolutions qui prolongent «naturellement » les thèmes fondateurs de l'anthropologie dans les cadres urbains et qui engendrent " potentiellement des effets considérables sur la constitution des savoirs de l'anthropologie en général, tant du point de vue de l'enquête que des concepts pour en rendre compte » (p. 16). Préoccupée par son urbanisation, l'anthropologie de la ville n'en reste pas moins générale : «ce n'est pas un autre nom pour l'anthropologie urbaine» (p.9), ni même une sous-catégorie de la discipline. Cette anthropologie est avant tout une "expérience localisée de vie, de découverte et de connaissance, tout comme un citadin est de Marseille, de Cali, [...] parce que c'est là qu'il vit, qu'il apprend et se socialise » (p. 20). En outre, l'enquête urbaine (multi)située permet d'en révéler les enfermements, les tensions, les innovations autant que les rencontres. C'est donc le processus de chaque ville qui se trouve au centre de la réflexion de l'anthropologie qui nous est proposée.

Dans deux de ses précédents ouvrages² ${ }^{2}$ l'auteur abordait déjà ce vaste sujet ainsi qu'une grande partie des thématiques connexes. Si ceux-ci avaient pu sembler déstruc-

\footnotetext{
${ }^{2}$ Michel Agier, L'invention de la ville : banlieues, townships, invasions et favelas, Paris, Éditions des Archives contemporaines, 1999 et Michel Agier, Esquisses d'une anthropologie de la ville, Louvain-la-Neuve, Éditions Academia-Bruylant, 2009.
} 
turés, lacunaires et souffraient peut-être d'avoir voulu brasser trop large, cette Anthropologie de la ville se révèle quant à elle particulièrement cohérente et convaincante. Conceptuellement, elle parvient à réconcilier l'anthropologie et la ville : le constat d'incompatibilité est dépassé et les apports spécifiques de la discipline sont assumés. Pragmatiquement, on ne pourra s'empêcher d'adhérer à l'urgence de renouveler la discipline et ses outils ainsi qu’à la nécessité de contribuer à une Anthropologie de la ville. Car, en transparence des argumentaires principaux, l'auteur nous rappelle assez clairement le rôle de contre-pouvoir des sciences sociales, cela sans trébucher dans d'indéfectibles et pusillanimes considérations épistémologiques au sujet d'une prétendue objectivité du chercheur. Difficile en effet de parcourir l'ouvrage sans y lire des échos flagrants avec l'actualité de ces derniers temps ; sans penser à des exemples concrets, quotidiens, vécus. L’anthropo-logique est partout ; l'« ailleurs » est à proximité... ce qui fournit en passant une généreuse poignée de grains à moudre à l'anthropologie du proche! Enfin, on ne peut que saluer cette démarche « aux frontières de l'achevé, de l'établi, de l'ordonné, du centre et du dominant » (p.30), encourageant l'étude - certes audacieuse - du changement, de l'instable ou encore des tensions permanentes et omniprésentes entre mobilité et immobilité. En résumé, cet ouvrage constitue une base solide et enthousiasmante en faveur d'une anthropologie renouvelée et actuelle : celle du chaos, des entre-deux, des interstices et des intervalles. Bref, une anthropologie prospective des centralités de demain. 